\title{
Poetiken des Todes bei Elfriede Jelinek
}

Die im zweiten Kapitel erörterte autobiografische Sterbeliteratur präsentiert eine literarische Bearbeitung von Sterbeprozessen: Tödlich erkrankte Schriftsteller:innen, die aufgrund einer medizinischen Diagnose eine begrenzte Lebenszeit vor sich haben, schreiben über die Erfahrung ihres eigenen Sterbens und bezeugen damit auch die Wucht und Monstrosität, welche die unmittelbare Konfrontation mit dem eigenen Sterben bedeutet. Wie dort erläutert, sind diese Artikulationen nicht zuletzt mit einem Willen nach (sprachlicher) Gestaltung des Lebensendes verbunden.

Eine andere Perspektive auf das Sterben präsentieren literarische Texte aus der Warte Hinterbliebener, die den Verlust eines Partners, eines Elternteils oder eines Kindes schildern und reflektieren. Dies hat in der Gegenwartsliteratur ebenfalls Konjunktur, wie Texte von David Grossmann über seinen im Libanonkrieg gefallenen Sohn, Naja Marie Aidt über den Tod ihres Sohnes, Joan Didion über den Tod ihres Ehemannes, Julian Barnes über den Verlust seiner Ehefrau, Melitta Breznik über das Sterben ihrer Mutter oder Zsuzsa Bánk über das Sterben ihres Vaters zeigen. ${ }^{1}$ Daneben sind fiktionale literarische Auseinandersetzungen mit Sterben und Tod an der Tagesordnung (als Beispiele der Gegenwartsliteratur vgl. etwa den fiktiven Sterbebericht von James Hannah oder den Roman über Sterbebegleitung von Susann Pásztor). ${ }^{2}$

Die unterschiedlichen literarischen Modi verdeutlichen, wie groß gegenwärtig die Reichweite literarischer Sterbe- und Todesreferenzen ist. Unabhängig von der jeweiligen thematischen Fokussierung - z. B. auf den Sterbeprozess, auf die institutionelle Versorgung von Sterbenden oder auf die Trauer über den Verlust - liegt der Mehrwert solcher literarischer Texte in der betont individuellen Artikulation von Ängsten, Nicht-Wissen, Ungewissheit, Schmerz etc. Derartige individuelle Wahrnehmungen und Affekte kommen in der Fachliteratur über das Lebensende aus der Gesundheitsforschung,

1 David Grossmann: „Der Frühling ist hier so kurz“ (Lied 2011); Naja Marie Aidt: When Death Takes Something from You Give It Back. London 2019 [dän. OA Har døden taget noget fra dig så giv det tilbage, 2017]; Joan Didion: Das Jahr magischen Denkens. Berlin 2006 [engl. OA The Year of Magical Thinking, 2005]; Julian Barnes: Lebensstufen, Teil 1. Köln 2015 [engl. OA Levels of Live, 2013]; Melitta Breznik: Mutter. Chronik eines Abschieds. München 2020; Zsuzsa Bánk: Sterben im Sommer. Frankfurt/M. 2020.

2 James Hannah: Das Alphabet der letzten Dinge. Köln 2016 [engl. The A to Z of You and Me, 2015]; Susann Pásztor: Und dann steht einer auf und öffnet das Fenster. Köln 2017. 
Psychologie, Geriatrie und Theologie kaum zur Sprache. Auch in den alltäglichen Gesprächen zwischen medizinischem Personal und Patient:innen ist dafür zumeist kaum Raum; allenfalls bleiben sie therapeutischen Gesprächssituationen vorbehalten.

Eine weitere, sehr spezifische Form literarischer Todesreferenz praktiziert die österreichische Schriftstellerin und Nobelpreisträgerin Elfriede Jelinek. Die Auseinandersetzung mit Tod, Sterbenmüssen und Gestorbensein durchzieht ihr Werk geradezu leitmotivisch mit anhaltender Dringlichkeit. Die Todesbezüge manifestieren sich dabei insbesondere durch Figuren, die zwischen Leben und Tod mäandern wie Vampire und Gespenster. Aber auch Opfer des Nationalsozialismus oder verstorbene historische Persönlichkeiten werden von der Autorin erweckt, um noch einmal zum Sprechen anzusetzen. Vor allem bringt Jelinek dabei verschwiegene Tote zum Sprechen: Personen, die zu Lebzeiten kaum eine Stimme hatten oder deren leidvolle Geschichten nicht bearbeitet werden konnten. Gerade deshalb treten sie nun wieder an die Oberfläche, so dass sich Jelineks Literatur auch als Hort der Toten gestaltet.

Anders als bei der autobiografischen Sterbeliteratur kommen dabei keine individuellen Affekte angesichts von Sterben und Tod auf. Überhaupt bieten Jelineks Texte kaum einen Resonanzraum für eine Besinnung auf das eigene physische Sterben. Vielmehr eröffnen sie den Blick auf anhaltende tödliche, insbesondere frauen- und fremdenfeindliche Gewalt- und Machtmechanismen, die zum Tod von immer neuen Einzelpersonen, Gruppen oder Communities führen. ${ }^{3}$ Diese spezifische, gesellschaftspolitisch geprägte Reichweite literarischer Todesreferenz wird in diesem Kapitel näher betrachtet.

\section{Über den Tod und das Untote: Reden, Essays, Statements}

Im Lauf der Jahre kommentiert Jelinek ihre literarischen Auseinandersetzungen mit dem Tod in Reden und Essays verschiedentlich unter poetologischen und programmatischen Gesichtspunkten. In ihrer Rede zum Literaturnobelpreis („Im Abseits“, 2004) formuliert sie erstmals eine eigentliche Poetik des Todes als literarisches Verfahren. Im Zentrum der Nobelvorlesung steht die Arbeit mit und an der Sprache. In diesem Zusammenhang kommt Jelinek auf die Toten zu sprechen und hält dabei fest, dass sie gleichsam unter Zwang stehe, diese wahrnehmen zu müssen: „Es gibt einfach zu viele Tote, auf die

3 Vgl. Moire Mertens: „Untote, Zombies und VampirInnen. Die Kritik der Bio-Macht in Elfriede Jelineks Texten“. In: Elfriede Jelinek: Begegnungen im Grenzgebiet (hg. von Natalie Bloch und Dieter Heimböckel). Trier 2014, S. 39-55. 
ich schauen muss." Geknüpft wird dieser Blick auf die Toten an den Verlust von Sprachkontrolle: ,je deutlicher diese Aufforderung zum auf sie, die Toten, Schauen in mir ertönt, umso weniger kann ich auf meine Worte achten."

Die Autorin stellt ihr Sprechen anderen Stimmen zur Verfügung, ja fast scheint es, als agiere sie als literarisches Medium, so wie hellsichtige Medien sich für den Kontakt mit Verstorbenen anbieten: „Da kommt einer, der schon gestorben ist, und der spricht zu mir, obwohl das für ihn nicht vorgesehen ist. Er darf das, viele Tote sprechen jetzt mit ihren erstickten Stimmen. ${ }^{45}$

Der wenige Monate später veröffentlichte Essay „Wir müssen weg“ (2005) konzentriert sich auf die literarische Wiederkehr von Toten, von denen sich die Autorin nach Belieben eine oder am liebsten viele Stimmen ausleiht, „damit sie sprechen, als wären sie ich, als wäre ich sie“. Dabei nehmen die Toten die Gestalt von Projektionsflächen an, denen die Autorin „das, was gewesen ist, und sogar das, was nicht gewesen ist, in den verfallenden Leib hineindichten kann, wobei die nicht gedichtet sind, sondern ausrinnen, ausrinnen nach wie vor und wie immer, bis nichts mehr da ist, außer Staub und Erde.“6

Die eigentümliche Dialektik des ausrinnenden Hineindichtens macht die Fragilität des Verfahrens deutlich. Andernorts spricht Jelinek davon, dass wir uns an „ungesicherten Leitfäden“ von Toten „entlangtasten“, was für ihre eigene Auseinandersetzung mit den Toten charakteristisch sei:

Wie kann man Erinnertes fassen, an das man sich selbst gar nicht erinnern kann, weil man es nicht erlebt hat? Erlebt haben es andre, sehr viele, die meisten von ihnen sind tot. An ihren, der Toten ungesicherten Leitfäden müssen wir uns entlangtasten und aufpassen, dass sie uns nicht aus den Händen rutschen oder wie Spinnweben zerreißen. ${ }^{7}$

In einem weiteren programmatischen Text mit dem Titel „Ich als TotenAusgräberin“ (2012) macht Jelinek den Kontext der Nachgeschichte des Nationalsozialismus explizit und spricht von einem literarischen Verfahren gegen „das Begrabensein von Schuld“. Dabei zielt sie insbesondere auf die „gespenstische Geschichte“ Österreichs: „[Ü]berall, wo man gräbt“, kämen „Knochen aus dem Boden“, und deshalb habe sie, so sagt die Autorin, „die Untoten immer

4 Elfriede Jelinek: „Im Abseits“. Nobelvorlesung 2004. Online unter: http://www.nobelprize. org/nobel_prizes/literature/laureates/2004/jelinek-lecture-g.html (letzter Zugriff: 23.11.2021).

5 Ebd. (letzter Zugriff: 23.11.2021).

6 Elfriede Jelinek: „Wir müssen weg. Eine wütende Polemik auf den Tod“. In: Cicero. Magazin für politische Kultur 10 (2005). Online unter: https://www.cicero.de/kultur/elfriedejelinekwir-müssen-weg/37119 (letzter Zugriff: 23.11.2021).

7 Elfriede Jelinek: „Mit den Augen von Toten schauen“. In: Verein Zeitgeschichte Museum Ebensee (Hg.): Retrospektive. 2o Jahre Geschichtsarbeit. Ebensee 2008, S. 4. 
schon als Metapher für die österreichische Geschichte gesehen.“ Und: „Es ist vielleicht eine Art Besessenheit, mit der ich mich immer wieder auf die Opfer der Nazizeit stürze, als ob ich sie wieder ausgraben könnte.“8

Angesichts der vielfältigen Verfahren, welche die literarische Sprache als Aufzeichnungs- und Vermittlungsinstrument für Totenstimmen birgt, ist es nachvollziehbar, dass Jelinek in einem Interview von 2007 gar sich selbst in den Kontext der Untoten rückt: „Ich bin nicht tot, aber ich empfinde mich als eine lebende Tote, weil ich - auf Grund meiner psychischen Erkrankung, über die ich nicht weiter sprechen möchte - eben nicht leben kann, nicht reisen kann, Menschen nicht ertrage. ${ }^{\text {99 }}$ Tatsächlich wird damit eine eigene neurotische Disposition geltend gemacht, deren Verfestigung nicht zuletzt auf öffentliche Anfeindungen gegen Jelinek zurückzuführen sein dürfte, die damit selbst in den Kontext der von der Autorin literarisch bearbeiteten gesellschaftlichen (Ab-)Tötungsprozesse treten.

\section{Die literarischen Figuren und der Tod}

Exemplarisch für Jelineks Literarisierung von Totenstimmen ist der 1995 erschienene Roman Die Kinder der Toten, der sich eingehend mit der Verdrängung des Nationalsozialismus und dessen Nachgeschichte in Österreich auseinandersetzt. Das umfangreiche Buch wird verschiedentlich als Opus magnum bezeichnet, in welchem die dominierenden literarischen Themen und Verfahren der Autorin kulminieren.

Die Rahmengeschichte des Romans spielt in der Fremdenpension Alpenrose in der Steiermark. Protagonisten sind drei untote Figuren, die nach ihrem Tod wiederkehren und gesellschaftliche gewalttätige Mechanismen anprangern. Die verschwiegene Kontinuität der nationalsozialistischen Geschichte in Österreich wird ebenso kritisiert wie die Politik der FPÖ, die katholische Kirche, der Sport in der Alpenwelt u. a. m. Dabei eröffnen die Figuren nicht nur eine Sphäre zwischen Tod und Leben, sondern auch zwischen den Zeiten: Sie agieren in verschiedenen Jahrzehnten und lassen auf diese Weise fließende Übergänge zwischen den 1940er Jahren und der Gegenwart entstehen, in denen die Präsenz nationalsozialistischer Praktiken in Österreich sichtbar wird. Der

8 Elfriede Jelinek: „Ich als Toten-Ausgräberin“. In: Pia Janke (Hg.): Jelinek[Jahr]Buch 3. Wien 2012, S. 17-19, Zitate S. 17 f.

9 Elfriede Jelinek im Interview mit Rose-Marie Gropp. „Dieses Buch ist kein Buch“. In: Frankfurter Allgemeine Zeitung, 17. April 2007. Online unter: http://www.faz.net/aktuell/feuilleton/ buecher/elfriede-jelinek-dieses-buch-ist-kein-buch-1434778.html (letzter Zugriff: 23.11.2021). 
Roman, pünktlich zu den 5o-Jahr-Gedenkfeiern zum Kriegsende veröffentlicht, ist ein wesentlicher literarischer Beitrag zu den (Nicht-)Debatten über die Kontinuität des Faschismus in Österreich.

Mit dem Kunstgriff der untoten Figur bringt die Autorin das Gedächtnis der Toten zum Sprechen. Dabei fluten die Toten die Lebenden, das Jenseits flutet das Diesseits, die Vergangenheit flutet die Gegenwart. Die wiederkehrenden Toten lassen das gesellschaftlich Abgedrängte wieder aufbrechen und kritisieren dieses Abdrängen gleichzeitig als Gewaltakt. Gerade in der begrifflichen Verwischtheit von ,untot ${ }^{\prime}$ - halb lebend, halb tot, nicht ganz lebendig, nicht ganz tot - liegt die kaum fassbare Sprengkraft dieser Figuren.

Zu Beginn ihrer literarischen Karriere inszenierte Jelinek Untote insbesondere im Kontext ihrer Geschlechterbefragung. So treten etwa im Theaterstück Krankheit oder Moderne Frauen (1984) die beiden weiblichen Vampire Emily und Carmilla als unerledigte Relikte der Literaturgeschichte in der Gegenwart auf. Die Namen verweisen auf die Autorin Emily Brontë sowie auf die Protagonistin von Sheridan Le Fanus Vampir-Novelle Carmilla (1872). Ihre Vampirexistenz - sie sind Pseudotote, weder tot noch lebend - steht dabei auch für die symbolische Ortlosigkeit der Frau. Bereits hier spricht Emily thesenhaft aus, was zwanzig Jahre später in Jelineks Nobelpreisrede poetologisch entfaltet wird: „Die Toten sollen lesbar sein.“"10

Auch das mehrteilige, zwischen 1998 und 2003 verfasste Theater-Projekt Der Tod und das Mädchen. Prinzessinnendramen bringt historische Frauenfiguren als Untote zum Sprechen. Hier kehren u. a. Schneewittchen, Jackie Kennedy, Sylvia Plath und Ingeborg Bachmann wieder. Sie rezensieren ihre eigenen Leben und Todesarten gleichsam posthum und kommentieren dabei auch die Bilder, die man sich von ihnen gemacht hat. Wieder zielt Jelinek auf Erinnerung und Kritik an gesellschaftlichen Praktiken ab, welche zur gewaltvollen Konstruktion von Weiblichkeitsbildern beitragen. An diesen Bildern sind die prominenten Figuren zugrunde gegangen, und als Wiedergängerinnen ihrer selbst bringen sie die Bilder erneut auf.

\section{Testamentarisches, dichterische Hinterlassenschaft}

Die Beispiele verdeutlichen, dass Jelineks literarische Todes-Figurationen ohne autobiografische Referenzen auskommen. Sie verweisen vielmehr auf ein gewaltsames ,Zu-Tode-gebracht-Werden' anderer und schaffen so einen literarischen Resonanzraum für gesellschaftliche Mechanismen, die

\footnotetext{
$10 \quad$ Elfriede Jelinek: Theaterstücke. Reinbek bei Hamburg 1992, S. 231.
} 
kontinuierlich, strukturell und systematisch mit (Ab-)Tötung einhergehen. Indem die Autorin sich jedoch, wie erwähnt, 2007 selbst als ,lebende Tote bezeichnete, deutet sie doch eine persönliche Involviertheit an.

Eine ebenso spielerische Auseinandersetzung mit dem eigenen Tod bietet sie um dieselbe Zeit im Kapitel $5 \mathrm{~g}$ des nahezu 1000-seitigen InternetFortsetzungs-Romans Neid. Privatroman (2007/2008), in dem die Frage des dichterischen Nachlasses verhandelt wird. Die Autorin präsentiert eine Art literarisches Pseudo-Testament, mit dem sie die Unwägbarkeiten urheberrechtlicher Verfügungen ad absurdum führt: Sie entwirft das Bild einer funktionalen Literatur, die materiell in Form von bedrucktem Papier überlebt, welches geschreddert wird und zum Häuserbau verwendet werden kann. Die Dichtung kommt so als Dämmstoff zum Einsatz, als (Ab-)Dichtungsmaterial, das je nach Bedarf Wasser, Wärme oder Kälte abhalten kann. ${ }^{11}$

Das Bild entbehrt nicht der Ironie: Dichtung als Wohlgefühl, als Schutzwall gegen alles, was von draußen hereinkommen könnte. Laut Bärbel Lücke hinterlegte Jelinek damit „öffentlich ihr dichterisches privates Vermächtnis, ihr Testament."12 Voraussetzung für diese Lesart ist der Umstand, dass Neid gerade im fünften Kapitel eine höchstmögliche Durchlässigkeit der Grenzen zwischen Autorin, Erzählerin und Protagonistin inszeniert. Das geht so weit, dass die Ich-Erzählerin sich über die Ähnlichkeit zwischen ihr und der Protagonistin auslässt. Die Unmöglichkeit einer Grenzziehung zwischen Fiktion, Autobiografie und „Tagebuch“, wie es im Text selbst heißt, ist damit auf die Spitze getrieben; aufgrund der hochgradigen literarischen Stilisierung ist es unmöglich festzulegen, welche Art von „Vermächtnis“ bzw. „Testament“ vorliegt. Ein juristisch umsetzbares Statement bietet das Romankapitel mit Sicherheit nicht - ganz abgesehen davon, dass der ausschließlich online publizierte Text von Jelinek jederzeit abgeändert werden kann, was sich die Autorin auch ausdrücklich vorbehält. ${ }^{13}$

Lücke erwähnt darüber hinaus, die Autorin habe „tatsächlich testamentarisch verfügt", dass die Originale ihrer Werke geschreddert werden sollen. Diese Aussage belegt sie mit der Zitation persönlicher E-Mails, die sie 2007 von Jelinek erhalten hat. Dabei habe die Autorin ihr mitgeteilt: „Die Originale werden ja geschreddert" / "Grad war mein Anwalt da, und ich habe mein Testament hinterlegt und dafür gesorgt, daß meine Urheberrechte an

11 Elfriede Jelinek: Neid. Privatroman. 2007/2008. Online unter: http://elfriedejelinek.com/ fneid-5b4.htm (letzter Zugriff: 23.11.2021).

12 Bärbel Lücke: www.todsuende.com. Lesarten zu Elfriede Jelineks „Neid“. Wien 2009, S. 188.

13 Elfriede Jelinek im Interview mit Rose-Marie Gropp (2007): http://www.faz.net/aktuell/ feuilleton/buecher/elfriede-jelinek-dieses-buch-ist-kein-buch-1434778.html (letzter Zugriff: 23.11.2O21). 
den Dingen, die zu Lebzeiten nicht erschienen sind (mein bald geschredderter Nachlass bzw. Vorlass), gewahrt bleiben, in seiner Kanzlei.."14 Auch solche E-Mail-Benachrichtigungen können kaum ernsthaft als testamentarische Verfügung gelten, und Jelinek erwähnt ja, dass das eigentliche Testament inklusive Regelung der Urheberrechte am Nachlass in den Händen des Anwalts liegt. Janke/Kovacs immerhin kommen aufgrund der Darlegungen von Bärbel Lücke zu dem Schluss, dass sich die Philolog:innen keine Hoffnungen machen sollten auf Dokumente, die Jelineks Arbeitsprozesse belegen (z. B. mit handschriftlichen Korrekturen der Autorin oder Kommentaren ihres Lektors). ${ }^{15}$

Der nicht fassbare Charakter wird durch die Diffusion von fiktionalen, postalischen und juristischen Äußerungen unterstrichen. Die Regelung der literarischen Hinterlassenschaft von Elfriede Jelinek verbleibt damit in einem Bereich zwischen Imaginationen, Fiktion und Realität. Die Aussagen sind diffus, scherzhaft, nicht fassbar. Der Umgang mit der Nachlassfrage bleibt also ungebunden, er ist an keine letzte Verbindlichkeit geknüpft und darüber hinaus an einem Nachlassleben im Netz orientiert, welches ohnehin unkontrollierbar sein wird.

\section{Jelinek: Autorschaft zum Tode}

In der Sekundärliteratur zu Jelinek ist häufig von Textkörpern und Sprachflächen die Rede. Mit solchen Worten fasst man jene literarischen Verfahren der Autorin, die sich jeglichem Identifikationsangebot verweigern, kaum erkennbare Handlungsstränge präsentieren und Erzählung nur noch fragmentarisch aufblinken lassen. Verbunden ist dies mit einer überbordenden Intertextualität und obsessiven Verwendung von Zitaten. Diese dienen, anders als das gewöhnliche Zitat, nicht dazu, den Deutungsstatus des auktorialen Sprechens zu stärken. Im Gegenteil wird ein ,eigenes Sprechen' durch die vielen Intertexte und Zitate geradezu verschüttet, zerrüttet, aufgelöst. Besonders augenfällig wird dies etwa im erwähnten Roman Die Kinder der Toten, bei dessen Lektüre man sich als Leserin phasenweise nur schwer zurechtfindet; man erhält kaum stabile Informationen, ist desorientiert und wird ständig übersprochen. Solches Verfahren trägt dazu bei, den Geschichts- und Verbrauchscharakter der Sprache offenzulegen: Sprache ist immer schon vor einem da gewesen, sie ist alt, benutzt, verbraucht, eine Hinterlassenschaft aus früheren Zeiten, ein Erbe

14 Lücke (2009): www.todsuende.com, S. 88.

15 Pia Janke und Teresa Kovacs: Publikationsformen und Werküberlieferung. In: Pia Janke (Hg.): Jelinek-Handbuch. Stuttgart 2013, S. 27-33. 
verstorbener Menschen. Andere haben sie verwendet und abgeschliffen, man ist in ihr (wie auf Erden) lediglich eine Zeitlang zu Gast, während der man sich ihrer annimmt, sich in sie einschreibt und sie auch wieder abgibt.

Jelinek betreibt ihre Poetik des Todes als eine Poetik des An- und Abwesenden, des Verschwindens und Wiederhervorkommens, des Daseins und NichtDaseins. In diesem Kontext ist auch die Präsentation der Nobelpreisrede zu sehen, die die Autorin nicht leibhaftig in Stockholm hielt, sondern per Videobotschaft einspielen ließ. Die Poetik ist - sei es in der literaturbetrieblichen Medienlandschaft oder im literarischen Text selbst - offen, anfällig, prekär, denn sie operiert am Rande des Untergangs, sie ist stets vom Verschwinden bedroht (thematisch, medial, poetologisch). Sie ist riskant und im poetologischen Sinne radikal, weil sie beständig und konstitutiv an die Wurzeln der eigenen Sprachexistenz rührt: Sie attackiert und demontiert die Fundamente der traditionellen Autorinstanz, indem sie diese bis zur Unkenntlichkeit zerspricht.

Mit dieser Poetik des Todes unterscheidet sich Jelineks Literatur wesentlich von den eingangs angeführten verschiedenen Modi der Sterbe- und Todesartikulationen: Sie weist keine vordergründigen autobiografischen Bezüge auf, sie fokussiert nicht das Individuum mit all seinem Schmerz, seinen Fragen und Ängsten angesichts von Tod und Sterben. Es geht weder um die individuelle Vergänglichkeit noch um die literarische Erkundung spiritueller Dimensionen oder einer möglichen Form eines Jenseits, und es geht auch nicht um eine literarische Darstellung psychologischer Trauerprozesse. Vielmehr problematisiert die Autorin gesellschaftspolitische Gewalt, welche systematische psychoökonomische Unterdrückung bewirkt und zu realen physischen Toden von immer neuen Personen und Gruppen führt: Die Referenz auf Tod und Tote bei Elfriede Jelinek konstituiert sich durch die kontinuierliche literarische Bearbeitung und Sichtbarmachung dieser Gewalt und legt dabei insbesondere deren strukturellen Aspekte frei. 